\title{
An Improved Wavelet-based Watermarking Method Using the Mathematical Morphology
}

\author{
Kasemsuk Sepsirisuk, Kiyoaki Atsuta, and Shozo Kondo \\ Graduate School of Engineering, Tokai University, Kanagawa, Japan \\ Email:kskasems@kmitl.ac.th
}

\begin{abstract}
In this paper, we propose an improved waveletbased watermarking method using the mathematical morphology. The wavelet coefficients of host image are firstly selected as the significant coefficients by a statistical predetermined threshold. The mathematical operations are then performed to tame some small perturbations on the selected coefficients caused by the manipulations or attacks. Moreover, the pixel-wise masking model which exploits the characteristics of Human Visual System is employed for improving watermark invisibility and robustness. The experimental results verify that our method is more robust and effective than the existing method.
\end{abstract}

Keyword: Image watermarking, pixel-wise masking, mathematical morphology.

\section{Introduction}

In recent years, the rapid development of Internet introduces a new set of challenging problems concerning security. One of the important problems is to discourage the unauthorized duplication of digital data. Digital watermarking has been proposed as an effective tool for protecting, authenticating, and tracking the copyrighted contents [1].

The watermarking techniques may be classified by a type of detector. One technique is a non-blind technique which requires an original image to detect a watermark [2]. The other technique is a blind technique which does not require the original image [3-5]. Among the techniques which do not require the original image for detection, Dugad et al. [3] embed a watermark into the most significant DWT coefficients of image. But their technique does not embed the watermark into an enough number of coefficients. So their technique is vulnerable to attacks such as sharpening. Barni et. al [4] shows an impressive performance of their work, in which the pixel-wise masking according to the Human Visual System (HVS) is proposed. However their algorithm adds the watermark into only the three largest detail subbands, which gives the opportunity to remove it easily to the attackers. Based on Barni's method, Xie and Shen [5] proposed an improved pixel-wise masking model and a new attack-resilient embedder. Their pixel-wise masking model can describe the HVS characteristic more effectively and accurately. They choose the high attack-resilient blocks in the wavelet domain to convey the watermark. An attack-resilient block is determined by using the Mean Square Error (MSE) between the block and the corresponding block after applying the JPEG compression to the original image. However, the selected blocks usually locate at the smooth areas. Thus, their concept contradicts the rule of watermarking that the watermark should be embedded into the human perceptual insensitive areas such as the edges and textured areas rather than smooth areas. Following the above consideration, an improved robust watermarking using the mathematical morphology is proposed in this paper.

Our paper is organized as follows. Section 2 describes the details of the improved robust watermarking. Section 3 provides the experimental results. Finally, section 4 concludes this paper.

\section{Improved Robust Watermarking}

In order to achieve the goals of robustness and imperceptibility, a watermark should be hidden in the resilient parts of host image with the high energy as much as it does not introduce visible distortion. Fortunately, with the excellent spatial-frequency localization properties of DWT, such goals can be achieved easily. In our method, the watermark is embedded into the called robust significant coefficient structure (RSCS), which is made to be resilient to various kinds of attack. This structure is needed to 
synchronize the embedded positions of coefficients at a detection process.

\subsection{Watermark Embedding}

An image to be watermarked is first decomposed with the 9/7 bi-orthogonal wavelets [7] into $L$ levels. Let $I_{l}^{\theta}$ be the coefficients in a subband at a resolution level $l=1,2, \cdots, L$ with an orientation $\theta \in\{a, h, v, d\}$ as illustrated in Fig.1. A watermark $X$, which is a sequence of binary numbers $\{-1,1\}$, is generated by the pseudorandom number generator with a secret key $K$. The coefficients in the RSCS are only chosen to convey the watermark; the RSCS is represented by a switching matrix $S_{l}^{\theta}(i, j) \in\{1,0\}$. Let $W_{l}^{\theta}(i, j)$ be a perceptual weighting function, which is corresponding to the local sensitivity of image to noise. The coefficients are then modified by using the formula

$\hat{I}_{l}^{\theta}(i, j)=I_{l}^{\theta}(i, j)+\alpha \cdot S_{l}^{\theta}(i, j) \cdot W_{l}^{\theta}(i, j) \cdot X_{l}^{\theta}(i, j),(1)$

where $\alpha$ is a global factor controlling the watermark energy.

\subsection{Perceptual Weighting Function}

There are various papers, which propose applications of the perceptual model of HVS. Most of them classify the sensitivity of HVS to noise into three major factors as follows $[4,5,7]$.

1. Luminance sensitivity: The HVS is less sensitive to noise in those areas of the image, where brightness is either high or low.

2. Spatial masking effect: The HVS is less sensitive to noise in the high textured areas.

3. Frequency sensitivity: The HVS is less sensitive to noise in the high frequency bands.

C. H. Chou et al. [7] proposed a definition of the Just Noticeable Difference (JND), which is first computed in image domain and then derived for frequency domain. On the other hand, based on a work of Barni et al. [4], Xie and Shen [5] proposed the improved pixel-wise masking model from a characteristic of wavelet coefficients. We use the Xie and Shen's model as the weighting function to evaluate the effectiveness.

\subsection{Robust Significant Coefficient Structure (RSCS)}

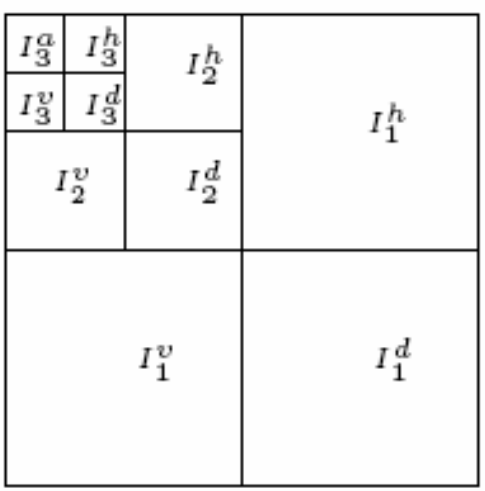

Figure 1. DWT with 3 levels decomposition.

In blind detection, without knowledge of host image, the synchronization is an important key to the detector response performance. Consider an attack, which manipulates wavelet coefficients that some nonembedded coefficients in a watermarked image are chosen as the embedded coefficients or vise versa. These occurrences yield the poor detector response.

In our method, the RSCS is constructed by the following procedures:

1. The wavelet coefficients are classified with a given threshold to obtain a binary "significance map", which is composed of all coefficients larger than the threshold. This threshold is content-adaptive defined equal to the sum of mean and standard deviation of magnitude of coefficients in the level $L$ detail subbands.

2. Morphological filtering is performed on the significance map to keep only the connected coefficients and to discard the isolated coefficients.

3. Furthermore, the morphological dilation $[8,9]$ is then performed.

A window size in step 2 and a structure element in step 3 are $3 \times 3$. The dilation produces an enlarged set of the original one by adding some nearby coefficients.

\subsection{Watermark Detection}

A watermark detection process of the proposed method is a blind method, which is performed without referring to the original image. The traditional correlation coefficient [10] is used to decide the presence of a watermark.

At the beginning of process, $S_{l}^{\theta}(i, j)$ is estimated from the testing image by using the same procedure of embedding process. The coefficients $I^{\prime}$ of testing image, which are corresponding to $S_{l}^{\theta}(i, j)=1$, are then used for computing the correlation coefficient $Z_{c c}$ as follows: 


$$
Z_{c c}=\frac{\tilde{I} \cdot \tilde{X}}{|\tilde{I}||\tilde{X}|},
$$

where $\tilde{I}$ and $\tilde{X}$ are obtained by subtracting out the means of the coefficients $I^{\prime}$ of testing image and watermark $X$, respectively.

\section{Experimental Results}

To objectively evaluate the performance of proposed method, we compare our RSCS with Xie and Shen's attack-resilient embedder [5] using the $512 \times$ 512 grayscale images. The level $L$ of DWT decomposition is 4 . We set the other conditions for the both methods as similar as possible. We use the same perceptual weighting function $W$, but different global factors $\alpha$, which are chosen to guarantee the same watermark strength, i.e., the PSNR between the watermarked images and original image is same in the both methods ( $38.80 \mathrm{~dB}$ for Lena).

The watermarked images are shown in Fig. 2. Although the PSNRs are same, there are block artifacts in Xie and Shen's result, since his switching matrix is determined by block-wise. Furthermore, his attackresilient embedder selects the block in the smooth areas rather than textured areas (Fig. 3 (b)). Since the MSE between the block of smooth area and its corresponding compressed block is usually low. So there are many visible noises in the smooth areas such as the face, while our method doesn't produce such noises.

Due to the stability of RSCS, which is constructed by applying the morphological operations, almost of coefficients in the estimated switching matrix in detection process is included in the embedding switching matrix. Fig. 3 (c) and (d) illustrate the similarity of the RSCS in embedding process and in detection process after applying the JPEG compression $(\mathrm{Q}=10)$.

The robustness comparisons of the both methods after applying various attacks of the famous watermark evaluation software, Stirmark (version 3.1) [11], are shown in Fig. 4 (a). A set of attacks includes as follows:

1. No attack

2. $2 \times 2$ Median filtering

3. $3 \times 3$ Median filtering

4. $4 \times 4$ Median filtering

5. FMLR

6. $3 \times 3$ Gaussian filtering

7. $3 \times 3$ Sharpening.

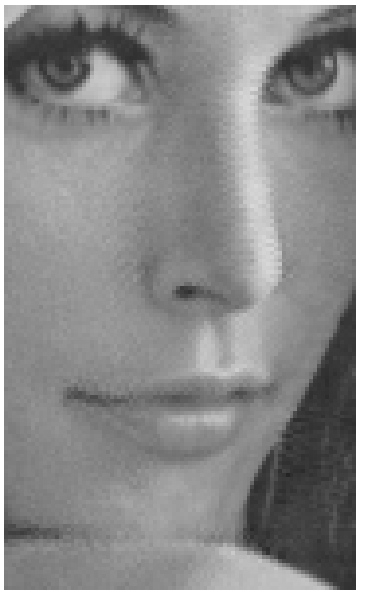

(a) Xie and Shen's method



(b) Proposed method
Figure 2. The watermarked images.

In Fig. 4 (a), the both methods have the similar results except the results of FMLR attack and Sharpening attack. Xie and Shen's method is vulnerable to FMLR, which removes the noises in frequency domain. Our method has a low robustness against sharpening, because the magnitude of wavelet coefficients became higher. Thus, many non-embedded coefficients are included in the RSCS. However, if we increase a threshold for selecting the significant coefficients, the detector response will be similar to Xie and Shen's method.

The evaluations of JPEG and JPEG2000 are shown in Fig. 4 (b) and (c), respectively. Note that our results are represented with solid line, while Xie and Shen's results are represented with dash line. Obviously, the proposed method is robust than Xie and Shen's method. Note that the Xie and Shen's attack resilient embedder is designed based on the JPEG attack $(\mathrm{Q}=10)$, so their method is very robust against that attack.

\section{Conclusions}

In this paper, we proposed an improved waveletbased watermarking method, in which the pixel-wise masking is taken into account. The mathematical morphology is applied to the significance map to improve the stability of significance map even after applying the image manipulation. Our watermarking method is clearly more effective than Xie and Shen's method. 


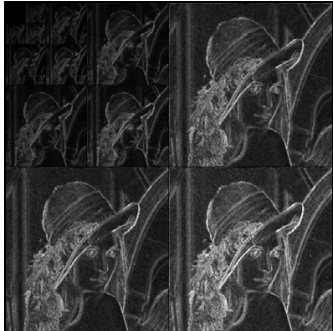

(a)



(b)

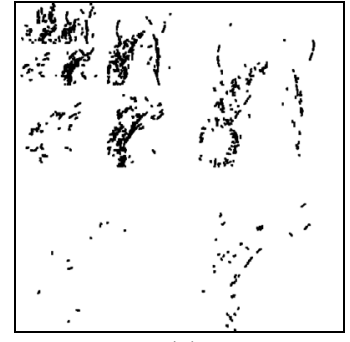

(c)

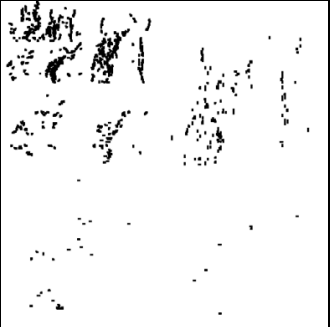

(d)

Figure 3. (a) Pixel-wise masking, (b) switching matrix in [5], (c) and (d) are the proposed switching matrixes of embedding and detection with JPEG compression $(Q=10)$, respectively. The black pixels in (b), (c), and (d) are corresponding to $S=1$.

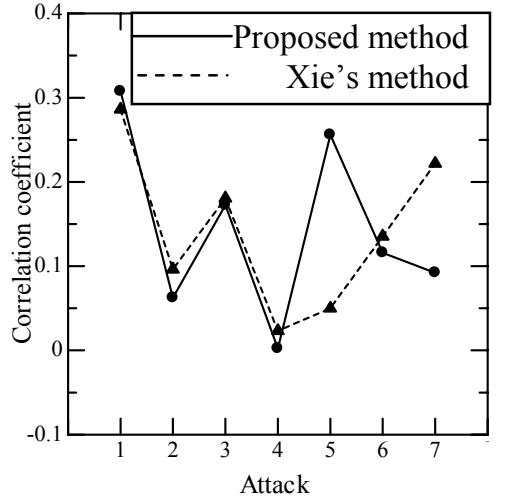

(a) Stirmark



(b) JPEG

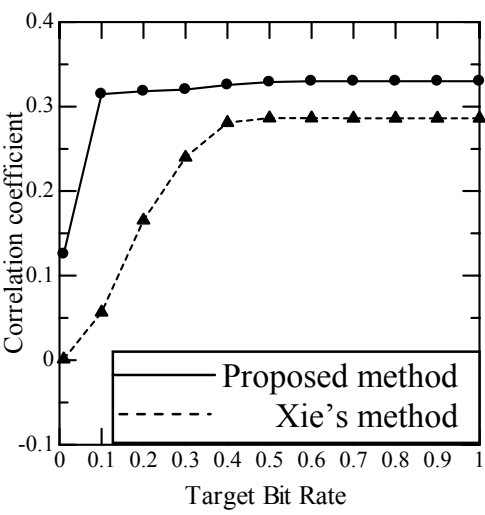

(c) JPEG2000

Figure 4. Comparison of robustness against attacks.

\section{References}

[1] F. Hartung and M. Kutter, "Multimedia watermarking techniques," Proc. of IEEE, Vol. 87, pp.1079-1107, 1999.

[2] R. B. Wolfgang, C. I. Podilchuk, and E. J. Delp, "Perceptual watermarking for images and video," Proc. of IEEE, Vol. 87, No. 7, pp. 1108-1126, 1999.

[3] R. Dugad, K. Ratakonda, and N. Ahuja, "A new wavelet-based scheme for watermarking images," Proc. of IEEE ICIP, pp. 357-372, 1998.

[4] M. Barni, F. Bartolini and A. Piva, "Improved WaveletBased Watermarking Through Pixel-Wise Masking", IEEE Trans. Image Process., vol.10, no.5, May 2001.

[5] Gui Xie and Hong Shen, "Toward Improved WaveletBased Watermarking Using the Pixel-Wise Masking Model", Proc. of IEEE ICIP, Vol. 1, pp. 689-692, 2005.

[6] A. Skodras, C. Christopoulos, and T. Ebrahimi, "The JPEG 2000 still image compression standard", IEEE Signal Proc. Mag., vol. 18, no. 5, pp. 36-58, Sept. 2001.
[7] C. H. Chou and K. C. Liu, "A perceptually tuned subband image coder based on the measure of justnoticeable-distortion profile," IEEE Trans. on Circuits and Systems for Video Technology, Vol. 5, Issue 6, pp. 467476, 1995.

[8] S. D. Servetto, K. Ramchandran and M. T. Orchard, "Image Coding Based on a Morphological Prepresentation of Wavelet Data", IEEE Trans. Image Process., vol.8, no.9, pp.1161-1174, Sep. 1999.

[9] Q. Sun and S. F.Chang, "Semi-fragile image authentication using generic wavelet domain features and ECC", Proc. of IEEE ICIP, Vol. 2, pp. 901-904, 2002.

[10] I. J. Cox, M. L. Miller, and J. A. Bloom, Digital Watermarking, Morgan Kaufmann Publishers, 2002.

[11] F. A. P. Petitcolas. "Watermarking schemes evaluation", IEEE Signal Proc. Mag., vol. 17, no. 5, pp. 58-64, September 2000. 